\title{
An extra-domiciliary method of delivering entomopathogenic fungus, Metharizium anisopliae IP 46 for controlling adult populations of the malaria vector, Anopheles arabiensis
}

Dickson W Lwetoijera ${ }^{1,2^{*}}$, Robert D Sumaye ${ }^{1}$, Edith P Madumla', Deogratius R Kavishe ${ }^{1}$, Ladslaus L Mnyone ${ }^{1,3,4}$, Tanya L Russell ${ }^{1,5}$, Fredros O Okumu ${ }^{1,6}$

\begin{abstract}
Fungal biopesticides have the potential to significantly reduce densities of malaria vectors as well as associated malaria transmission. In previous field trials, entomopathogenic fungus was delivered from within human dwellings, where its efficacy was limited by low infection rates of target mosquitoes, high costs of spraying fungus inside houses, and potential public health concerns associated with introducing fungal conidia inside houses. Here we have demonstrated that Metarhizium anisopliae IP 46, delivered within an extra-domiciliary odor-baited station (OBS), can infect and slowly-kill a high proportion of the wild adult malaria vector, Anopheles arabiensis which entered and exited the OBS. This study, carried out in rural Tanzania, showed that by using a concentration of $3.9 \times 10^{10}$ conidia/ $\mathrm{m}^{2}$, more than $95 \%$ of mosquitoes that flew in and out of the OBS died within 14 days postexposure. At least $86 \%$ infection of mosquito cadavers was recorded with a significant reduction in the probability of daily survival of exposed An. arabiensis in both treatments tested: low quantity of conidia (eave baffles plus one cotton panel; $H R=2.65, P<0.0001$ ) and high quantity of conidia (eave baffles plus two cotton panels; $H R=2.32$, $P<0.0001)$. We conclude that high infection rates of entomopathogenic fungi on wild malaria vectors and possibly significant disruption of malaria transmission can be achieved if the fungus is delivered using optimally located outdoor odor-baited stations.
\end{abstract}

\section{Findings}

Despite the widely documented potential of entomopathogenic fungi, such as Metarhizium anisopliae and Beauveria bassiana to infect and kill adult disease-transmitting mosquitoes [1-3], it remains questionable how feasible and effective this technology would be in real life situations. Specific concerns include: 1) lack of knowledge regarding the efficacy of different fungal strains under field conditions and 2) how to best deliver the fungi so as to achieve maximum infection rates while minimizing public health concerns associated with introducing fungal conidia inside houses [2]. Using the entomopathogenic fungus, $M$. anisopliae IP46, this study assessed the potential of using odor-baited

\footnotetext{
* Correspondence: dwilson@ihi.or.tz
'Biomedical and Environmental Thematic Group, Ifakara Health Institute, PO

* Correspondence: dwilson@ihi.or.tz
${ }^{1}$ Biomedical and Environmental Thematic Group, Ifakara Health Institute, PO Box 53, Ifakara, Tanzania
}

stations located away from human dwellings as a means of addressing these concerns.

The production and viability testing of $M$. anisopliae IP46 fungal conidia for the experiment has previously been described by Mnyone et al. [1]. The stock and working-suspension of fungal conidia were formulated in mineral oil [4,5], using Enerpar (Enerpar $\mathrm{M002}^{\circledR}, \mathrm{BP}$ South Africa Ltd) and Shellsol (Shellsol ${ }^{\oplus}$ T, United Kingdom) at a ratio of $1: 1$, to obtain the required oil viscosity. The fungal formulation was applied to the exposure surfaces at a concentration of $3.9 \times 10^{10}$ coni$\mathrm{dia} / \mathrm{m}^{2}$, using a hand-held pressure sprayer (Minijet ${ }^{\circ}$, SATA, Germany) set at a constant pressure of 2 bars. To achieve this, the nozzle of the spray gun was held $0.5 \mathrm{~m}$ away from and perpendicular to the surface sprayed. Each $23 \mathrm{ml}$ of working-suspension containing the required conidia concentration was applied evenly 
to a $1 \mathrm{~m}^{2}$ area [4]. The exposure surfaces were black cotton cloth made into eave-baffles (1.37 m length $\times$ $0.43 \mathrm{~m}$ width $)$ and panels $(1.3 \mathrm{~m}$ length $\times 1.1 \mathrm{~m}$ width $)$. The panels were treated on both sides to maximize contact surface area for mosquitoes once inside the OBS, whereas eave-baffles were treated only on one side, the upper side, to infect mosquitoes while entering the OBS. Untreated eave-baffles and panels were sprayed with oil the formulation alone. Both treated and untreated control surfaces were dried for 48 hours in separate rooms to avoid contamination, then wrapped in foil and stored at $4^{\circ} \mathrm{C}$ before being transferred to the field.

The field study was conducted in Lupiro Village $\left(8.384977^{\circ} \mathrm{S}\right.$ and $\left.36.670158^{\circ} \mathrm{E}\right)$, Ulanga District, in south east Tanzania, where the predominant malaria vector is An. arabiensis [6]. The fungus was disseminated in the field using odor-baited stations (OBS; Fig 1). The OBS is essentially a hut-shaped box made of canvas on a wooden framework. It measures $1.5 \mathrm{~m} \times 1.5 \mathrm{~m}$ and its highest point is $1.75 \mathrm{~m}$ from its wooden basement. On one side, it has a round operator entry point $(0.6 \mathrm{~m}$ diameter) fitted with a black cotton sleeve. In addition, it had four eave openings for mosquito entry or exit $(0.17$ $\mathrm{m} \times 1.4 \mathrm{~m}$ ). The entire inside of the device is lined with black cotton cloth except the floor which is covered with a plastic floor mat. The device may be used as a trap when fitted with interception exit traps (made of ultraviolent-resistant netting on a wire frame with a funnel-shaped entry point) or as contamination/killing station when incorporated with mosquito killing agents, in this case, Metarhizium anisopliae IP46. Development and initial field evaluation of this device has been reported elsewhere [7].

The OBS was baited with a synthetic mosquito lure, a blend consisting of carboxylic acids, ammonia and carbon dioxide. This lure was recently developed at Ifakara Health Institute $[6,8]$. To reduce costs and ensure ease of handling, one constituent of the synthetic lure, industrial $\mathrm{CO}_{2}$ gas was replaced with organic $\mathrm{CO}_{2}$, made from a cocktail of baker's yeast and sugar $[9,10]$. The mixture was prepared at least one hour before commencing the experiment to ensure that the yeast-sugar digestion process was already ongoing when the experiment started. The $\mathrm{CO}_{2}$ apparatus consisted of two separate plastic pots each having 500 grams of sugar and 3 litres water. Each pot contained a different amount of baker's yeast i.e. $80 \mathrm{~g}$ and $150 \mathrm{~g}$ of respectively. The yeast-sugar suspension inside both pots was adequately stirred before the pots were placed inside the OBS. This two-pot system, initially described by Saito et al [9] ensures a continuous supply of sufficient $\mathrm{CO}_{2}$ gas throughout the night. For the purposes of standardization in these experiments, the yeast-sugar preparations were replaced on a daily basis even though the gas supply was clearly never depleted after this period.

Three OBS located at $50 \mathrm{~m}$ apart in triangular setup were used. They were labeled as OBS 1 to 3 , and the set of three treatments were rotated within the OBS. For each OBS, two opposite eave openings were designated for mosquito entry and, to reduce egress, these points were fitted with black cotton cloth baffles [11]. When treated, the eave-baffles could potentially disseminate fungal conidia to mosquitoes when they land on it as they enter the OBS via the space $(0.05 \mathrm{~m})$ between the eave-baffle and the roof cover. The other two eave openings were fitted with exit traps (Fig 1A). In addition, inside each OBS, a maximum of two panels were hung vertically in the OBS and at $0.5 \mathrm{~m}$ from each other (Fig 1C). Treatment 1, used the lowest quantity of conidia consisting of two baffles and one panel treated with fungal conidia, while Treatment 2, used the highest quantity of conidia consisting of two baffles and two panels treated with fungi fitted inside the OBS. Lastly, Treatment 3, represented the untreated control where two oil-treated baffles and one oil-treated panel were fitted in the OBS. The experiment was conducted between October and November 2009 for 18 nights in two 9-night blocks, with a single treatment of panels and baffles for each block. During the experiment, the treatments were rotated daily between the different OBS using a $3 \times 3$ Latin square experimental design, as such the treatments were replicated 3 times per block.

A total of 300 live unfed An. arabiensis (untreated control $=101$, Treatment $1=100$, Treatment $2=99$ ) were sampled using aspirators from exit traps during 18 experiment nights, with maximum of $20 \mathrm{An}$. arabiensis sampled per night per treatment. No mosquitoes were observed to be resting inside of the OBS.

Ribosomal DNA species identification [12] was not performed, and instead all the collected An. gambiae complex mosquitoes were assumed to be $A n$. arabiensis; based on the evidence from Okumu et al. [7] which showed that 99\% of all An. gambiae collected from the same study area at the same time as this study were $A n$. arabiensis.

Daily survival and fungal infection status of individual mosquitoes were assessed in an improvised field insectary for 22 days. Mosquitoes were kept alive on $10 \%$ glucose solution in scratched plastic tubes $(0.027 \mathrm{~m}$ diameter $\times 0.115 \mathrm{~m}$ height), until death, after which their cadavers were prepared for fungal infection colonization. Cadavers were put in Petri dishes with a dry filter paper (to avoid growth of opportunistic fungi), and kept in a sealed humid chamber (container with a moistened towel) for fungal sporulation [5]. Hyphal growth on the cadavers, indicative of fungal infection, was observed at days 5-6 post incubation. Median survival 

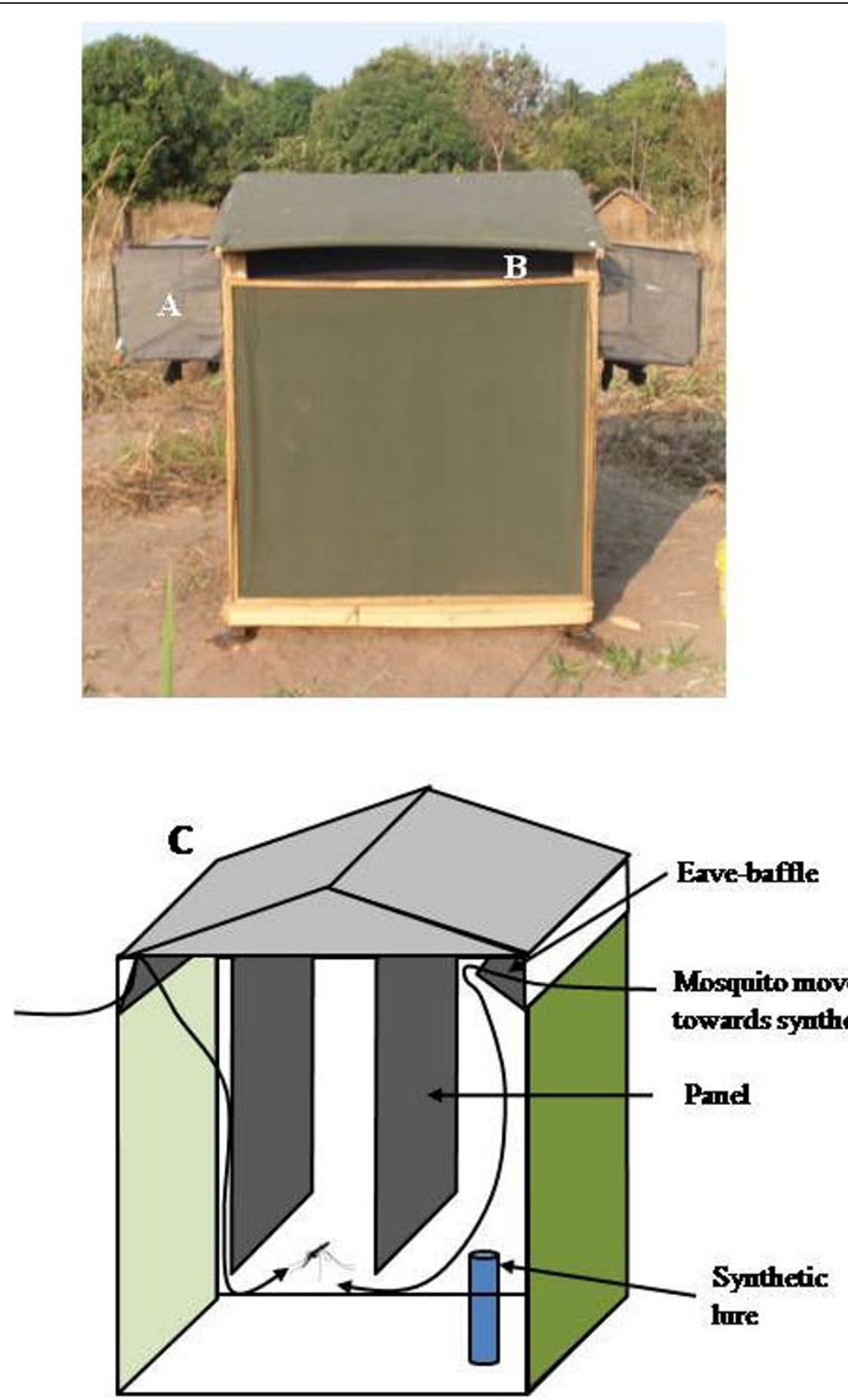

Figure 1 Picture of the odor-baited station fitted with (A) exit trap, (B) conidia treated baffles, and (C) schematic representation.

data were analyzed using Cox proportional hazards regression, to compute the hazard ratio (HR) and relative daily risk of dying for mosquitoes in treated groups compared to the daily risk of dying in the untreated control group, using the $R$ analysis software package V2.9.1.

When integrated into a lure and kill system using the OBS under field settings, Metarhizium anisopliae IP46 competently infected high proportions of the sampled wild female An. arabiensis. The percentage of mosquito cadavers from treated groups that sporulated was $85.7 \%$ in Treatment 1 and $86.1 \%$ in Treatment 2, while sporulation in the untreated control group was $9.3 \%$. It is not obvious what might have been the source of infection in controls but it is likely that since the OBS were only 50 $\mathrm{m}$ apart, a small number of mosquitoes may have 
successfully escaped from treated OBS and ended up inside untreated OBS.

The daily probability of survival of mosquitoes in the treated groups was significantly reduced compared to the untreated group $(P<0.0001$, Table 1, Fig. 2$)$. More than $95 \%$ mortality was observed in the treated groups by day 14 , whereas $30 \%$ of mosquitoes in the untreated control group were still alive at this time. One hundred percent mortality of mosquitoes in the treated groups and untreated group was achieved by days 16 and 22 respectively. The Mean Survival Time (MST) of An. arabiensis from the treated groups was 2 days and was five-times

Table 1 Median survival time (MST) of wild Anopheles arabiensis exposed to Metarhizium anisopliae IP46 in odor-baited-stations interception trap; differences were compared with Cox regression model. The hazard ratios (HR) indicate the relative risk of death in treatment groups compared with the untreated control.

\begin{tabular}{llll}
\hline Treatment & MST (IQR) & HR [95\% Cl] & $\boldsymbol{p}$ value \\
\hline Control & $10(2.8-14.3)$ & 1.00 & \\
Treatment 1 & $2(1.0-4.0)$ & $2.65[2.45-2.86]$ & $<0.0001$ \\
Treatment 2 & $2(1.0-5.5)$ & $2.32[2.16-2.49]$ & $<0.0001$ \\
\hline
\end{tabular}

Notes: MST = median survival time; IQR = inter quartile range; $\mathrm{HR}=$ hazard ratio; $\mathrm{Cl}=$ confidence limit. lower than the MST of the untreated control mosquitoes, which was 10 days. The probability of mosquito death was twice as likely in Treatment $1(\mathrm{HR}=2.65, P<0.0001)$ and Treatment $2(\mathrm{HR}=2.32, P<0.0001)$ than in the untreated group $(\mathrm{HR}=1$, Table 1$)$.

To achieve complete interruption of malaria transmission, development of new tools for vector control, including entomopathogenic fungi, needs to be prioritised. In future, the efficacy of current vector control measures, insecticide treated nets (ITN) and indoor residual spraying (IRS) could be lost due to pyrethroid insecticide resistance [13] and or changes in the resting and feeding behaviour of relevant vectors [14]. Nevertheless, it has been also demonstrated that entomopathogenic fungi, a slow-killing bio-pesticide [5] can work synergistically with exiting vector control measures; either by killing pyrethroid-resistant mosquitoes or increasing their susceptibility to insecticides and targeting different life-history parameters $[15,16]$.

In this study we demonstrated that by incorporating Metarhizium anisopliae IP46 in odor-baited stations away from human dwellings, wild populations of female An. arabiensis can be lured and killed within 1-5 days. At day 14, which is the maximum time for maturation to the infective stage of malaria Plasmodium $[17,18]$,

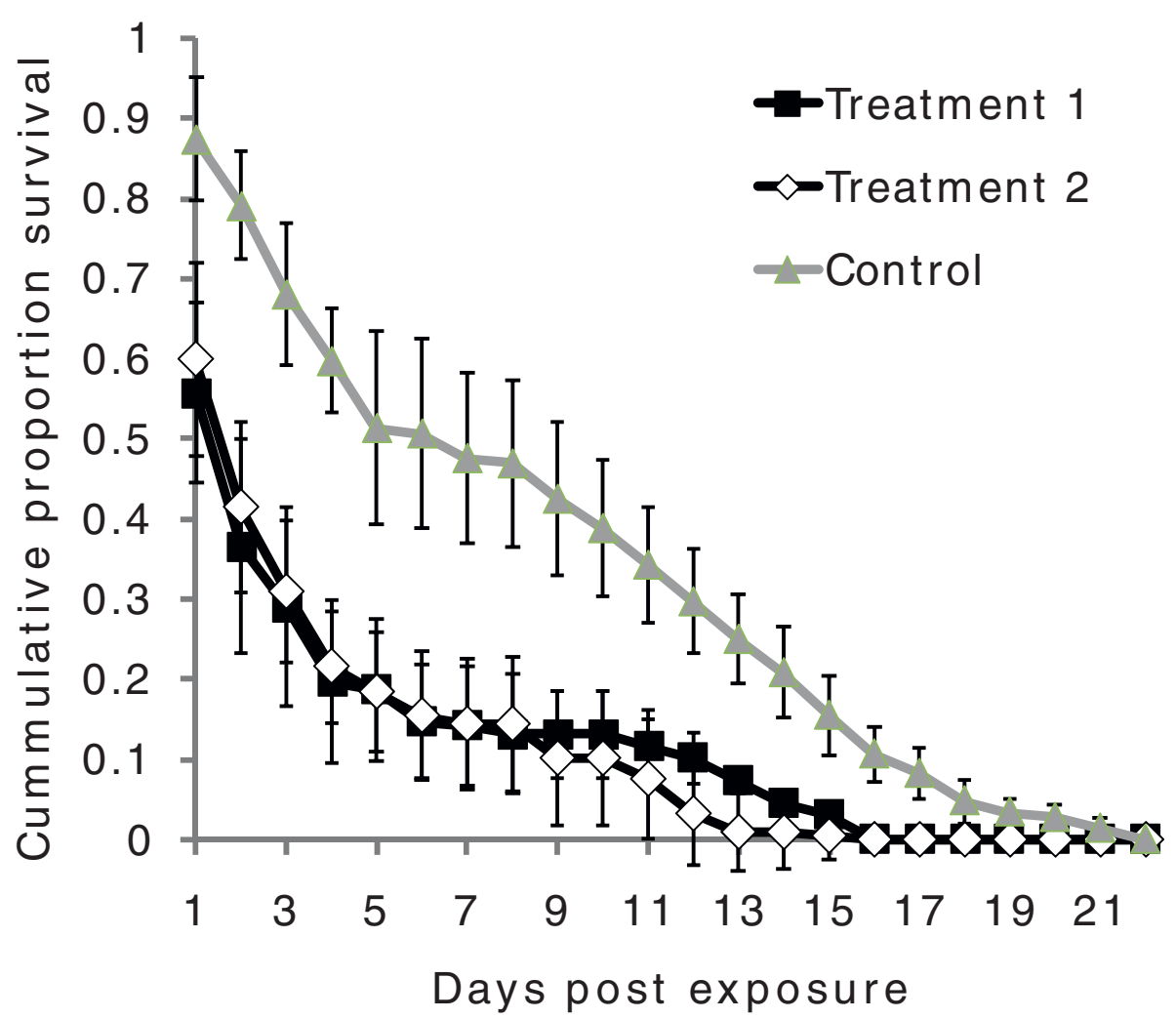

Figure 2 Survival of wild Anopheles arabiensis after exposure to Metarhizium anisopliae IP 46 conidia in odor-bated station 
$>95 \%$ mortality had occurred in treated groups. These results correspond with laboratory and field findings done elsewhere, $[1,2,4,5]$. Although mosquito mortality is delayed, it is faster than the extrinsic incubation period of malaria parasites and therefore able to bring about significant reductions in transmission control [15], and facilitate the possibility for genes of infected mosquitoes to be passed to sequential generations, thus weakening the selection pressure for resistance development $[19,20]$.

The level of infection in the treated groups was almost the same despite the difference in number of treated surfaces. This suggests that infection levels may not have been dependent only on the number of treated surfaces, but rather on whether the mosquitoes come into contact with the surfaces. Therefore, the impact of either baffles or panels alone or both on the level of infection may need to be investigated further.

The current intra-domiciliary interventions, ITNs and IRS remain the most effective means for malaria vector control [13]. However these interventions are not sufficient to meet the goal of malaria elimination as their benefits are limited to indoor biting mosquitoes while neglecting outdoor biting populations [14]. We therefore propose that effective use of odor-baited stations as the delivery method for entomopathogenic fungi may be considered as an option to target mosquitoes while they are outdoors and thus address the proportion of malaria transmission not directly controllable using ITNs and IRS [21].

Though fairly bulky, the organic $\mathrm{CO}_{2}$ production system used in these experiments was not only cheaper than the industrial carbon dioxide gas used in our previous tests [7], but it was also more readily available and easier to handle. Nevertheless, for sustainable applicability and efficacious community-wide adoption of this novel technology it will be necessary to: 1) continue research towards impregnation mechanisms that would ensure that the fungi remain persistent for long periods in the field, 2) optimize the synthetic lure and associated $\mathrm{CO}_{2}$ production system and 3) develop appropriate geolocation models to guide implementation of the strategy in real life operations.

Finally, this OBS technique presents an opportunity to not only increase infection rates beyond that which have been recorded inside human houses [2] but also to increase the likelihood of acceptance of this technology by avoiding to introduce conidia within human occupied houses which, though unproven, could be very costly and could have ethical and medical consequences. The system therefore is a potential outdoor means through which village-wide application of the bio-pesticide or other potent insecticides can be delivered for effective interruption of malaria transmission, but it also represents opportunities to address the potential public health concerns associated with introducing fungal pathogens inside people's houses.

\section{Acknowledgements}

The authors are grateful to Novatus Lipindi and Monica Mpingwa for helping with fungal production, supervising and monitoring field activities. Many thanks to Christian Luz for providing original fungal strain, $M$. anisopliae IP46 Instituto de Patologia Tropical e Saúde Pública, Universidade Federal Goiás, Goiânia, Brazil. We also thank the three anonymous reviewers of this paper for their very constructive comments and suggestions. This project was funded by a Grand Challenges Exploration Award (Grant code 15214), from the Global Health Discovery program of the Bill and Melinda Gates Foundation.

\section{Author details}

${ }^{1}$ Biomedical and Environmental Thematic Group, Ifakara Health Institute, PO Box 53, Ifakara, Tanzania. '2Department of Zoology and Wildlife Conservation, University of Dar es Salaam, PO Box 35091, Dar es Salaam, Tanzania. ${ }^{3}$ Pest Management Center, Sokoine University of Agriculture, PO Box 3110, Morogoro, Tanzania. ${ }^{4}$ Laboratory of Entomology, Wageningen University \& Research Centre, PO Box 8031, 6700 EH, Wageningen, The Netherlands. ${ }^{5}$ Vector Group, Liverpool School of Tropical Medicine, Liverpool, L3 5QA, UK. ${ }^{6}$ London School of Hygiene and Tropical Medicine, Keppel Street, London WC1E, 7HT, UK.

\section{Authors' contributions}

Conceived and designed the experiments: DWL, RDS and FOO. Performed the experiments: DWL, EPM. Analyzed the data: TLR, DWL. Wrote the paper: DWL, FOO, RDS, DRK, TLR, and LLM.

\section{Competing interests}

The authors declare that they have no competing interests.

Received: 20 February 2010 Accepted: 16 March 2010

Published: 16 March 2010

\section{References}

1. Mnyone LL, Lwetoijera DW, Russell TL, Lyimo NI, Kirby MJ, Luz C: First report of Metarhizium anisopliae IP 46 pathogenicity in adult Anopheles gambiae s.s. and An. arabiensis (Diptera; Culicidae). Parasite \& Vectors 2009, 2:59.

2. Scholte EJ, Ng'habi K, Kihonda J, Takken W, Paaijmans K, Abdulla S, Killeen GF, Knols BGJ: An entomopathogenic fungus for control of adult African malaria mosquitoes. Science 2005, 308:1641.

3. Scholte EJ, Knols BGJ, Takken W: Infection of the malaria mosquito Anopheles gambiae with the entomopathogenic fungus Metarhizium anisopliae reduces blood feeding and fecundity. J Invertebr Pathol 2006, 91:43-49.

4. Mnyone LL, Kirby MJ, Lwetoijera DW, Knols BJG, Takken W, Russell TL: Infection of the malaria mosquito, Anopheles gambiae, with two species of entomopathogenic fungi: effects of concentration, co-formulation, exposure time and persistence. Malar J 2009, 8:309.

5. Blanford S, Chan BHK, Jenkins N, Sim D, Turner RJ, Read AF, Thomas MB Fungal pathogen reduces potential for malaria transmission. Science 2005, 308:1638.

6. Okumu F, Killeen G, Ogoma O, Biswaro L, Smallegange R, Mbeyela E, Titus E, Munk C, Ngonyani H, Takken W, Mshinda H, Mukabana WR, Moore SJ: Development and field evaluation of a synthetic mosquito lure that is more attractive than humans. PLOS ONE 2010, 5:e8951.

7. Okumu FO, Madumla EP, Alex JN, Lwetoijera DW, Sumaye RD: Attracting, trapping and killing disease-transmitting mosquitoes using odor-baited stations - The Ifakara Odor-Baited Stations. Parasites \& Vectors 2010, 3:12

8. Okumu F, Biswaro L, Mbeleyela E, Killeen G, Mukabana R, Moore S: Using nylon strips to dispense mosquito attractants for sampling the malaria vector Anopheles gambiae s.s.. J Med Entomol 2010, 47:274-282.

9. Saitoh Y, Hattori J, Chinone S, Nihei N, Tsuda Y, Kurahashi H, Kobayashi M: Yeast generated $\mathrm{CO}_{2}$ as a convinient source of Carbon dioxide for Adult mosquito Sampling. J Am Mosa Control Assoc 2004, 20:261-264. 
10. Oli K, Jeffery J, Vythilingam I: A comparative study of adult mosquito trapping using dry ice and yeast generated carbon dioxide. Trop Biomed 2005, 22:249-251.

11. Smith A, Hudson JE: A modification to an experimental hut to reduce mosquito eaves-egress. WHO 1972, 72.775:6.

12. Scott AJ, Brogdon GW, Collins HF: Identification of single specimens of the Anopheles gambiae complex by the polymerase chain reaction. Am J Trop Med Hyg 1993, 49:520-529.

13. WHO: World malaria report. Geneva 2009

14. Pates H, Curtis C: Mosquito behavior and Vector Control. Annu Rev Entomol 2005, 50:53-70.

15. Farenhorst M, Mouatcho JC, Kikankie CK, Brooke BD, Hunt RH, Thomas MB, Koekemoer LL, Knols BGJ, Coetzee M: Fungal infection counters insecticide resistance in African malaria mosquitoes. Proc Natl Acad Sci USA 2009, 106:17443-17447.

16. Hancock PA: Combining Fungal Biopesticides and Insecticide-Treated Bednets to Enhance Malaria Control. PLoS Comput Biol 2009, 5:11.

17. Talman AM, Domarle O, McKenzie FE, Ariey F, Robert V:

Gametocytogenesis: the puberty of Plasmodium falciparum. Malar J 2004, 3:24.

18. Beier JC: Malaria parasite development in mosquitoes. Annu Rev Entomol 1998, 43:519-543.

19. Read AF, Lynch PA, Thomas MB: How to make evolution-proof insecticides for malaria control. PLOS Biol 2009, 7:e1000058.

20. Thomas MB, Read AF: Can fungal biopesticides control malaria? Nat Rev Microbiol 2007, 5:377-383.

21. Bayoh MN, Mathias DK, Odiere MR, Mutuku FM, Kamau L, Gimnig JE, Vulule JM, Hawley WA, Hamel MJ, Walker ED: Anopheles gambiae: historical population decline associated with regional distribution of insecticide-treated bed nets in western Nyanza Province, Kenya. Malar J 2010, 9:62.

doi:10.1186/1756-3305-3-18

Cite this article as: Lwetoijera et al: An extra-domiciliary method of delivering entomopathogenic fungus, Metharizium anisopliae IP 46 for controlling adult populations of the malaria vector, Anopheles arabiensis. Parasites \& Vectors 2010 3:18.

\section{Submit your next manuscript to BioMed Central and take full advantage of:}

- Convenient online submission

- Thorough peer review

- No space constraints or color figure charges

- Immediate publication on acceptance

- Inclusion in PubMed, CAS, Scopus and Google Scholar

- Research which is freely available for redistribution

Submit your manuscript at www.biomedcentral.com/submit
Biomed Central 